\title{
GW $(\Gamma)$ method without the Bethe-Salpeter equation for photoabsorption energies of spin-polarized systems
}

\author{
Tomoharu Isobe, ${ }^{1}$ Riichi Kuwahara, ${ }^{2}$ and Kaoru Ohno ${ }^{1, *}$ \\ ${ }^{1}$ Department of Physics, Yokohama National University, \\ 79-5 Tokiwadai, Hodogaya-ku, Yokohama 240-8501, Japan \\ ${ }^{2}$ Dassault Systèmes BIOVIA K.K., ThinkPark Tower, 2-1-1 Osaki, Shinagawa-ku, \\ Tokyo 141-6020, Japan \\ (Dated: May 14, 2018)
}

\begin{abstract}
The one-shot GW method beginning with the local density approximation (LDA) enables one to calculate photoemission and inverse photoemission spectra. In order to calculate photoabsorption spectra, one had to additionally solve the Bethe-Salpeter equation (BSE) for the two-particle (electron-hole) Green's function, which doubly induces the evaluation errors. It has been recently reported that the GW + BSE method significantly underestimates the experimental photoabsorption energies (PAEs) of small molecules. In order to avoid these problems, we propose to apply the $\mathrm{GW}(\Gamma)$ method not to the neutral ground state but to the cationic state to calculate PAEs without solving the BSE, which allows the rigorous one-to-one correspondence between the photoabsorption peak and the "extended" quasiparticle level. We applied the self-consistent LGW cluding the vertex correction to our method, and found that this method gives the PAEs of $\mathrm{B}, \mathrm{Na}_{3}$, and $\mathrm{Li}_{3}$ within the $0.1 \mathrm{eV}$ accuracy.
\end{abstract}

\section{INTRODUCTION}

Photoabsoprtion (PA) plays a decisive role in plenty of photo-related phenomena such as photosynthesis, photovoltaics cells, photocatalysts, photochemical reactions, photoinduced phase transitions, and so on. Photoabsoprtion energies (PAEs) have an important information on these phenomena. Physics behind PAEs for all wave length regions, if one ignores the vibronic effects, is that each PAE is quantum mechanically identified to the total energy difference between the ground state (GS) and an excited eigenstate (EES) with one electron excited from the GS of the Hamiltonian.

So far, we have three different first-principles approaches to calculate PAEs: (i) the quantum chemistry (QC) approaches such as the configuration interaction (CI) and coupled cluster (CC) methods,[1] (ii) time-dependent density functional theory (TDDFT),[2] and (iii) the GW + Bethe-Salpeter equation (BSE) approach.[3-7] The $\triangle \mathrm{SCF}$ method, which calculates the difference between the two total energies $E_{v}^{N}$ and $E_{G}^{N}$, has been commonly used in the QC approaches. They are very accurate but the computational cost required in the QC approaches scales as $\mathrm{O}\left(v N^{n}\right)$, where $v$ represents the number of the peak positions of the PA spectrum $[8,9]$ and $n$ represents the computational cost required in the GS calculation; for example, $n=6$ for multi-reference single and double CI (MRDCI).[8] (This is because $E_{v}^{N}$ is calculated after $E_{v-1}^{N}, E_{v-2}^{N}, \cdots, E_{G}^{N}$ in the orthogonality constrained method.[9]) Due to their heavy computational cost and low parallel efficiency, the QC approaches are not applicable to large molecules. TDDFT is probably the most economic option among the three, but the results strongly depend on a choice of the exchange-correlation functional. TDDFT is suitable for coarse estimation of large molecules.

*Electronic address: ohno@ynu.ac.jp
The GW + BSE approach is still heavy, but, thanks to the high parallel efficiency, its computational cost can be well dispersed. Recently, this approach has been applied to various materials.[10, 11] The approach is composed of two procedures; one is the GW approximation (GWA)[12, 13] to determine the quasiparticle (QP) energies, which represent the difference between the total energies of the $N$-electron GS and the $(N \pm 1)$-electron EES. They correspond to photoemission and inverse photoemission spectra (PES/IPES), where one electron is removed from or added to the $N$-electron GS. The energy gap $\varepsilon_{g}^{N}$ can be obtained by the difference between two QP energies, $\varepsilon_{\text {LUMO }}^{N}-\varepsilon_{\text {HOMO }}^{N}$, but this $\varepsilon_{g}^{N}$ is different from the PAE, $\Omega^{N}$. To calculate PAEs, one has to additionally treat the electron-hole, two-body problem by solving the BSE.[3-7] In this way, the method has to deal with not only the one-particle Green's function in the GW part but also the two-particle (electron-hole) Green's function in the BSE part, and doubly induces the evaluation errors. Indeed, it has been recently reported by several authors that the GW + BSE method significantly underestimates the experimental PAEs of atoms and small molecules.[14-16] The use of the Heyd-Scuseria-Ernzerhof (HSE) functional or the self-consistent GW calculation improves the results, but they are not perfect.[15, 17] This problem is difficult to solve in MBPT unless one uses a more sophisticated approach such as the self-consistent LGW + BSE approach.[16] In any approach solving the BSE, the resulting two-particle (electronhole) wave functions are the complicated linear combinations of the products of the electron and hole QP wave functions. Therefore, there is no one-to-one correspondence between each peak of the PA specturm (corresponding a $N$-particle EES) and the QP energy level (corresponding to a $N \pm 1$ particle EES).

In the present paper, we propose a completely different and very tractable approach, which allows the rigorous one-to-one correspondence between the PA peaks and the ("extended") QP levels,[18] although each peak height (intensity or oscil- 
lator strength) is not obtained. Our idea is to treat a cationic system instead of a neutral system and to consider the following three processes:

1. Consider a cationic state having one hole in the neutral GS and calculate the largest energy gain when one adds one electron to this cationic hole level to retrieve the neutral GS.

2. Calculate the second (or third, $\cdots$ ) largest energy gain when one adds one electron to the higher empty levels of this cationic state to construct the final PA state.

3. The first (or second, third, ...) PA peak energy is directly obtained as the difference between these two energy gains without any relaxation as shown in Fig. 1.

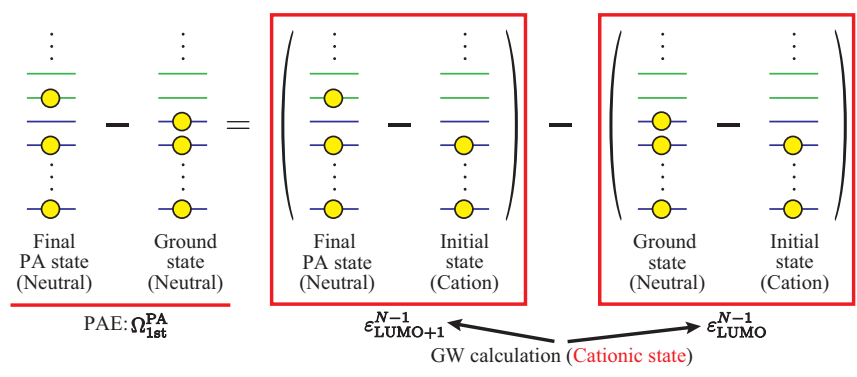

FIG. 1: The first peak PAE $\Omega_{1 \text { st }}^{\mathrm{PA}}$ is equal to the extended QP energy difference $\varepsilon_{\mathrm{LUMO}}^{N-1}-\varepsilon_{\mathrm{HOMO}}^{N-1}$ obtained by the GW calculation for the $(N-1)$-electron cationic GS. Here, $\varepsilon_{\mathrm{LUMO}}^{N-1}$ and $\varepsilon_{\mathrm{HOMO}}^{N-1}$ correspond, respectively, to the total energy differences between the final PA state and the initial cationic GS and between the neutral GS and the initial cationic GS.

A significant point of this method is that the two energy gains, i.e. the minus of the two "extended" QP energies, in the processes 1 and 2 can be obtained simultaneously by applying the standard GW method to the cationic system instead of the neutral system, and the last process 3 is just a simple subtraction of these two energies and does not require any relaxation procedure such as to solve the BSE. Therefore, it offers a very simple and elegant method which enables one to calculate the electron excitations from the HOMO level to the $\mathrm{LUMO}, \mathrm{LUMO}+1, \mathrm{LUMO}+2, \ldots$ levels just by a single GW calculation. (Note that some other PA peaks may correspond to the energy difference between the HOMO-1 level or, in general, the HOMO $-n$ level and the LUMO+1, LUMO+2, ... levels. To obtain such PA peaks, it is necessary to repeat the similar GW calculation by starting from the cationic EES with one hole at the HOMO-1 level or, in general, at the $\mathrm{HOMO}-n$ level. But anyway this is a simple and transparent task on the basis of the "extended QP theory."[18]) It allows one to identify the rigorous one-to-one correspondence between the PA peaks and the ("extended") QP levels. Since our new method requires the GW calculation only and does not require the $\mathrm{BSE}$, we named this as the " $\mathrm{GW}(\Gamma)$ method without BSE." This method is, without doubt, faster and simpler than the $\mathrm{GW}(\Gamma)+$ BSE method because the complicated BSE is no longer necessary. This method is named as " $\mathrm{GW}(\Gamma)$ " but our framework allows to replace the $G W(\Gamma)$ calculation to any other method which can calculate the ("extended") QP energies.

Although the present method is very powerful, some comments should be given here. It delivers excited state energies, but no oscillator strengths. This is crucial in the majority of applications, since experimental absorption spectra need to be understood and/or predicted. The BSE method needs not consider the fact that the excited state can be characterized by a single configuration (for example, a HOMO to LUMO transition), although it cannot specify the characterization of each transition. In addition, the vast majority of systems is spinunpolarized. For such systems the present approach requires a spin-polarized GW calculation (see below), while the standard GW+BSE approach can be done on closed-shell systems.

\section{BASIC THEORY}

In this section, we derive the $\mathrm{GW}(\Gamma)$ method without BSE to calculate PAEs and compare the method with the previous formalism. We apply the "extended" QP theory [18] to the $(N-1)$-electron system to obtain PAEs of the $N$-electron system.

Our purpose is to calculate the one-electron EES of the $N$ electron neutral system. One of the observable in such a single photo-excitation process is the vertical PAE. The corresponding physical quantity is the PAE defined as

$$
\Omega_{v}^{\mathrm{PA}}=E_{v}^{N}-E_{G}^{N},
$$

where $E_{v}^{N}$ and $E_{G}^{N}$ represent, respectively, the total energies of the $v$ th EES and the GS of the $N$-electron neutral system.

The simplest and clever way to calculate the PAEs is to consider the $(N-1)$-electron cationic system $\gamma$, in which the photoexcited electron is removed from the final PA state $v$. In this idea, the PAEs are obtained by comparing the total energy of this system $\left(E_{\gamma}^{N-1}\right)$ with those of the final state $\left(E_{v}^{N}\right)$ and the GS $\left(E_{G}^{N}\right)$. This $\gamma$ th eigenstate of the $(N-1)$-electron system plays a central role as the initial state. The $N$-electron neutral GS is retrieved if one electron is added to the one-electronmissing hole level in the initial cationic state $\gamma$, while the final photoabsorbed state $v$ is retrieved if one electron is added to the $v$ th empty level. In other words, the "extended" QP energies, $\varepsilon_{0}$ and $\varepsilon_{v}$, defined as the total energy differences between these states,

$$
\begin{aligned}
& \varepsilon_{0}=E_{G}^{N}-E_{\gamma}^{N-1}, \\
& \varepsilon_{v}=E_{v}^{N}-E_{\gamma}^{N-1},
\end{aligned}
$$

represent the energy gains when one electron is added to this initial $(N-1)$-electron cationic state $\gamma$. Such electron attachment energies of cations can be observed by the PES/IPES. Note that the former energy $\varepsilon_{0}$ is identical to the electron affinity (EA) of the cationic state $\gamma$ (or to the ionization potential (IP) of the neutral GS if $\gamma$ is the cationic GS), while the latter energy $\varepsilon_{v}$ corresponds to the $v$ th EA of the cationic state $\gamma$. Eq. (2b) becomes identical to Eq. (2a) when $v=0$ is the 
neutral GS. By using those energies, the PAEs can be simply obtained as

$$
\Omega_{v}^{\mathrm{PA}}=\varepsilon_{v}-\varepsilon_{0}
$$

without introducing any further relaxation.

The electron attachment energies $\varepsilon_{v}$ (including $\varepsilon_{0}$ ) can be calculated by solving the "extended" quasiparticle equation (EQPE):[18]

$$
h_{s}^{(1)} \phi_{v}(\boldsymbol{r}, s)+\int \Sigma_{s}\left(\boldsymbol{r}, \boldsymbol{r}^{\prime} ; \varepsilon_{v}\right) \phi_{v}\left(\boldsymbol{r}^{\prime}, s\right) d \boldsymbol{r}^{\prime}=\varepsilon_{v} \phi_{v}(\boldsymbol{r}, s),
$$

where $h_{s}^{(1)}$ and $\Sigma_{s}$ represent the one-body part of the Hamiltonian and the self-energy, respectively. The symbol $v$ may be either an occupied state or an empty state, but, in order to distinguish occupied and empty states, we will use $\mu$ for occupied states and $v$ for empty states in what follows. In usual cases, the equation is solved for the neutral GS of the $N$-electron neutral system, but, in the present case, it must be solved for the initial $(N-1)$-electron cationic state $\gamma$. Although the form of this equation is the same as the usual QP equation, we call it the EQPE to clarify that the initial system is not the $N$-particle neutral GS $\left|\Psi_{G}^{N}\right\rangle$ but the $(N-1)$-electron cationic state $\left|\Psi_{\gamma}^{N-1}\right\rangle$. For atoms and small molecules, we have confirmed that the EQPE can yield reasonable values of the extended QP energies[18] by using the GWA.

Eigenvalues and eigenfunctions of Eq. (4) are identical to

$$
\begin{gathered}
\varepsilon_{\mu}=E_{\gamma}^{N-1}-E_{\mu}^{N-2}, \\
\varepsilon_{v}=E_{v}^{N}-E_{\gamma}^{N-1},
\end{gathered}
$$

and

$$
\begin{gathered}
\phi_{\mu}(\boldsymbol{r}, s)=\left\langle\Psi_{\mu}^{N-2}\left|\psi_{s}(\boldsymbol{r})\right| \Psi_{\gamma}^{N-1}\right\rangle, \\
\phi_{v}^{*}(\boldsymbol{r}, s)=\left\langle\Psi_{v}^{N}\left|\psi_{s}^{\dagger}(\boldsymbol{r})\right| \Psi_{\gamma}^{N-1}\right\rangle
\end{gathered}
$$

where $\psi_{s}(\boldsymbol{r})$ and $\psi_{s}^{\dagger}(\boldsymbol{r})$ represent for annihilation and creation operators, respectively. Here, the level from which one electron is excited by the PA process of the neutral GS is labeled by the index $\gamma$, and the initial cationic state $\left|\Psi_{\gamma}^{N-1}\right\rangle$ is no other than the state with one electron missing at this $\gamma$ level in the neutral GS. If $\gamma=0$, then the PA energies correspond to the HOMO-electron excitations. In principle, there is no difficulty to consider the $\gamma>0$ cases (corresponding to the (HOMO-1)electron, (HOMO-2)-electron, ... excitations) as well. This enables us to know only the special sort of the PAEs associated with a particular orbital $\gamma$. As a matter of fact, the total energy, $E_{v}^{N}$, and total electron density, $n_{v}^{N}$, of the final $N$-electron state $v$ can be calculated via the simple relations

$$
\begin{gathered}
E_{v}^{N}=E_{\gamma}^{N-1}+\varepsilon_{v}, \\
n_{v}^{N}(\boldsymbol{r})=n_{\gamma}^{N-1}(\boldsymbol{r})+\phi_{\nu}^{*}(\boldsymbol{r}, s) \phi_{v}(\boldsymbol{r}, s),
\end{gathered}
$$

where $E_{\gamma}^{N-1}$ and $n_{\gamma}^{N-1}$ stand for the total energy and the total electron density of the $(N-1)$-electron cationic state $\gamma$.
Another merit of this method is that PAEs of spin-polarized systems can be calculated more easily than those of spinunpolarized systems, because PAEs of the spin-polarized systems can be calculated using the spin-unpolarized cations. Consider a spin-polarized system, in particular, a system whose spin multiplicity is doublet. Hamiltonian of the system is, of course, spin-polarized. However, by removing one electron from the HOMO level, the cationic system becomes spin-unpolarized, which makes the calculation easier. This simplification occurs in the case of $\gamma=0$, i.e., the excitation from the HOMO level to the LUMO $+n$ levels $(n=0,1,2 \ldots)$. In what follows, we restrict ourselves to the $\gamma=0$ case only, although this is not a necessary constraint in our framework.

The most important issue here is how to solve the EQPE (4). This issue is identical to the problem how to approximate the self-energy $\Sigma_{s}$. One of the popular approximation is one-shot GWA $\left(G_{0} W_{0}\right)$ but this method depends on exchangecorrelation functional of density functional theory (DFT) [19]. Self-consistent GWA has no functional dependence but usually overestimates the energy gap.[20, 21]

Ren et al.[22] treated the second-order screened exchange (SOSEX) and the renormalized single excitation (rSE). Their results for the binding energies of rare gas and copper clusters are in fairly good agreement with the experimental data; the mean absolute error is about $0.25 \mathrm{eV}$ for RPA + SOSEX and about $0.15 \mathrm{eV}$ for rPT2. Hung et al. [23] calculated IPs, EAs, and PAEs of aromatic molecules within the GW + BSE method. They included in their GW calculation a local density approximation (LDA)-derived vertex function $\left(G W \Gamma_{\mathrm{LDA}}\right)$. The first GW calculation (the GW $\Gamma^{1} @ \mathrm{HSE}$ method) was performed by Grüneis et al.,[24] who used GWTC-TC @HSE + single-shot vertex correction for the selfenergy with the static approximation. The results for the QP energies of various semiconductors and insulators are slightly better than those of GW $\mathrm{GC}^{\mathrm{TC}-\mathrm{TC}} @ \mathrm{HSE}$, but there is still at most $0.7 \mathrm{eV}$ difference from the experimental values. Maggio and Kresse [25] recently applied one-shot Hedin's scheme with the vertex function (GWT) for a set of small molecules. The agreement with the experimental IP is fairly good although not excellent.

There are two important steps toward the self-consistent GWT calculation. One is the problem of the energy dependence in the correlation part of the self-energy $\Sigma_{s}^{\mathrm{c}}=\Sigma_{s}-\Sigma_{s}^{\mathrm{x}}$. Shishkin et al. [21] proposed to linearize the energy dependence, but their method does not satisfy the Ward identity and the non-orthogonality problem remains in the resulting QP wave functions. Kuwahara et al.[26] proposed to linearize the energy dependence so as to satisfy the Ward identity and remove the non-orthogonality problem of the QP wave functions. The Green's function $G_{s}$ and the polarization function $P$ are renormalized in the LGW approach as follows: $G_{s}$ is renormalized using the lower triangular matrix $L$ as $\tilde{G}_{s}(\omega)=L^{\dagger} G_{s}(\omega) L$. This $L$ is defined by the Cholesky decomposition of $\Lambda$ as

$$
\Lambda=1-\left.\frac{\partial \Sigma_{s}(\omega)}{\partial \omega}\right|_{\omega=\omega_{0}}=L L^{\dagger}
$$

where $\omega_{0}$ means the energy around which the self-energy is 
expanded. It can be set, for example, at the mean value of the HOMO and LUMO eigenenergies. Renormalization of the polarization function $\tilde{P}$ is achieved by replacing the Green's function with the renormalized one: $\tilde{P}=-i \sum_{s} \tilde{G}_{s} \tilde{G}_{s}$. If the exact vertex function $\Gamma$ is known, then the GW $\Gamma$ approach gives the exact "extended" QP energies, but this is of course impossible. Kuwahara et al.[16] developed the self-consistent GW $\Gamma$ method by approximating the vertex function $\Gamma$ to first order in the dynamically screened Coulomb interaction $W$ (the $G W \Gamma_{W}$ method) or in the bare Coulomb interaction $v$ (the $\mathrm{GW}_{v}$ method). They showed that the linearization procedure mentioned above is applicable also to the self-consistent GW $\Gamma$ approach just by replacing the Green's function $G_{s}$ with its renormalized version $\tilde{G}_{s}$ (the $\mathrm{LGW}_{W}$ and $\mathrm{LGW}_{v}$ methods).[16] Their $\mathrm{LGW}_{W}+\mathrm{BSE}$ calculations produced excellent PAEs of $\mathrm{Na}, \mathrm{Na}_{3}, \mathrm{~B}_{2}$, and $\mathrm{C}_{2} \mathrm{H}_{2}$ within $0.1 \mathrm{eV}$ difference from the experimental values. In this paper, we use the (L)GW $\Gamma_{W}$ methods without BSE for cationic systems.

\section{CALCULATION RESULTS AND DISCUSSION}

\section{A. Computational detail}

We calculate the PAEs and IP of some spin-polarized isolated atoms and molecules by solving the extended QP equation (4) for the $(N-1)$-electron cationic GS, where one electron is removed from the HOMO level of the neutral GS. All calculations are performed using the all-electron mixed basis code, TOMBO, [16, 26, 27] which uses (numerical) atomic orbital (AO) and plane wave (PW) basis sets together. The minimal number of AOs including all occupied orbitals is used, although valence AOs are truncated within the non-overlapping atomic spheres. The cutoff energies of 11.06 (44.20) Ry, 14.44 (57.76) Ry, 2.18 (30.7) Ry, and 2.76 (38.9) Ry are used for $\mathrm{PW}, P$, and $\Sigma_{s}^{\mathrm{c}}$ (for the cutoff energy for $\Sigma_{s}^{\mathrm{x}}$ ), respectively, for $\mathrm{Al}, \mathrm{B}, \mathrm{Na}_{3}$, and $\mathrm{Li}_{3}$. The edge lengths of the face-enteredcubic unit cell are chosen as $18 \AA, 14 \AA, 18 \AA$, and $16 \AA$, respectively, for $\mathrm{Al}, \mathrm{B}, \mathrm{Na}_{3}$, and $\mathrm{Li}_{3}$. Moreover, the Coulomb spherical cutoff[3] is adopted to avoid the unphysical electrostatic interaction between periodic images. To apply this technique, a large enough unit cell is used. The bond lengths are $3.23,3.23$, and $5.01 \AA$ for $\mathrm{Na}_{3}$, and 2.76, 2.76, and $3.38 \AA$ for $\mathrm{Li}_{3}$. We use the plasmon-pole models $[12,28]$ and 800,1400 , 600, 600 levels, respectively, for $\mathrm{Al}, \mathrm{B}, \mathrm{Na}_{3}$, and $\mathrm{Li}_{3}$. For the $\mathrm{LGW} \Gamma$ calculations for $\mathrm{B}, \mathrm{Na}_{3}$, and $\mathrm{Li}_{3}$, we set $\omega_{0}$ at the value of $\mathrm{HOMO}+3 \mathrm{eV}$.

\section{B. Comparison of the results for IP/EA and $1^{\text {st }}$ PAE}

Theoretically, the IP of the neutral systems must be identical to the EA of the cationic systems if the same atomic geometry is assumed for neutral and cationic systems.[29] Here, the one-shot GW results for the IP of the neutral systems $(\mathrm{GW}(\mathrm{IP}))$ are listed in Table I together with the oneshot GW and self-consistent LGW results for the EA of the cationic systems (GW(EA) and LGWГ(EA)), all of which should be compared with the experimental data,[30-33] for $\mathrm{Al}, \mathrm{B}, \mathrm{Na}_{3}$, and $\mathrm{Li}_{3}$. If we compare $\mathrm{GW}(\mathrm{EA})$ with $\mathrm{GW}(\mathrm{IP})$, the former is in better agreement with the experimental value for $\mathrm{Al}$ (the difference between $\mathrm{GW}(\mathrm{EA})$ and the experiment is $0.1 \mathrm{eV}$ ), while $\mathrm{GW}(\mathrm{EA})$ is in worse agreement with the experiment for $\mathrm{Na}_{3}$ and $\mathrm{Li}_{3}$. Therefore, we performed the self-consistent $\mathrm{LGW}$ calculations for $\mathrm{B}, \mathrm{Na}_{3}$, and $\mathrm{Li}_{3}$. The resulting $\mathrm{LGW}(\mathrm{EA})$ is in excellent agreement with the experimental value for these systems; the difference between $\mathrm{LGW}(\mathrm{EA})$ and the experiment is about $0.1 \mathrm{eV}$.

TABLE I: GW results for the ionization potential (IP) of neutral systems (GW(IP)), GW and LGW of cationic systems (GW(EA) and LGWГ(EA)), and the corresponding experimental data[30-33] in units of eV

\begin{tabular}{lcccc}
\hline \hline atom/molecule & GW(IP) & GW(EA) & LGWГ(EA) & Experiment \\
\hline $\mathrm{Al}$ & 5.81 & 5.88 & - & $5.98^{\mathrm{a}}$ \\
\hline $\mathrm{B}$ & 8.45 & 8.45 & 8.20 & $8.30^{\mathrm{b}}$ \\
\hline $\mathrm{Na}_{3}$ & 4.24 & 4.35 & 4.21 & $4.08 \pm 0.05^{\mathrm{c}}$ \\
\hline $\mathrm{Li}_{3}$ & 4.24 & 4.44 & 4.12 & $3.97 \pm 0.05^{\mathrm{d}}$ \\
\hline \hline
\end{tabular}

${ }^{a}$ Ref. 30, ${ }^{b}$ Ref. 31, ${ }^{c}$ Ref. 32. ${ }^{d}$ Ref. 33,

Next, we show the GW + BSE (neutral) results and the GW (cation) results without $\mathrm{BSE}$ of the $1^{\text {st }} \mathrm{PAE}$ corresponding to the HOMO-LUMO transition for $\mathrm{Al}, \mathrm{B}, \mathrm{Li}_{3}$, and $\mathrm{Na}_{3}$ together with the experimental data [31,34-36] (the peak corresponding to the $\mathrm{A}$ band observed by experiments for $\mathrm{Na}_{3}[35]$ and $\mathrm{Li}_{3}$ [36]) in Table II. The GW + BSE (neutral) results significantly underestimate the experimental PAEs as anticipated (the mechanism of the underestimation is reported in the previous research[16]), while the GW (cation) results without BSE are in fair agreement with the experimental PAEs; the difference between the GW method without BSE and the experiment is about $0.2 \mathrm{eV}$. This means that the GW method without BSE for spin-unpolarized cation systems is simpler and more accurate than the GW + BSE method for spinpolarized neutral systems.

TABLE II: GW + BSE (neutral) and GW (cation) results without BSE (present work) for the $1^{\text {st }}$ PAE compared with the experimental data[31, 34-36] in eV

\begin{tabular}{lccc}
\hline \hline atom/molecule & GW + BSE & GW present work & Experiment \\
\hline $\mathrm{Al}$ & 2.94 & 3.23 & $3.14^{\mathrm{a}}$ \\
\hline $\mathrm{B}$ & 4.53 & 5.24 & $4.96^{\mathrm{b}}$ \\
\hline $\mathrm{Na}_{3}$ & 0.76 & 1.71 & $1.85^{\mathrm{c}}$ \\
\hline $\mathrm{Li}_{3}$ & 0.50 & 1.64 & $1.81^{\mathrm{d}}$ \\
\hline \hline
\end{tabular}

${ }^{a}$ Ref. 34, ${ }^{b}$ Ref. 31, ${ }^{c}$ Ref. 35. ${ }^{d}$ Ref. 36, 


\section{Comparison between the GW, GW $\Gamma$ and LGW $\Gamma$ methods without BSE and the QC methods for PAEs}

Here, we show the PAEs of $\mathrm{B}, \mathrm{Na}_{3}$, and $\mathrm{Li}_{3}$ calculated by the GW, GW and LGW compare them with the previous results of highly accurate QC calculations such as multi-configulation HF (MCHF), [37] MRDCI,[38, 39] full CI,[40] and estimated full CI[39] as well as the corresponding experimental data.[31, 35, 36, 41] We employed the self-consistent GW $\mathrm{G}$ approach and its linearized version (LGWT) as the beyond GW methods without BSE. All these data are listed in Tables III-V, which show the PAEs for the transitions designated in the left column.

Our self-consitent LGW results show excellent agreement with the experiments within $0.06 \mathrm{eV}$ in average and $0.10 \mathrm{eV}$ at maximum. This accuracy is enough to compare with other highly accurate QC calculations such as MRDCI and full CI.

TABLE III: B: GW, GW and LGW results (without BSE) for the PAEs compared with the previous MCHF results[37] and experimental data[31] in units of $\mathrm{eV}$

\begin{tabular}{|c|c|c|c|c|c|}
\hline Transition & GW & GWГ & LGWT & $\mathrm{MCHF}^{\mathrm{a}}$ & Experiment $^{\mathrm{b}}$ \\
\hline $2 s^{2} 2 p-2 s^{2} 3 s$ & 5.24 & 4.39 & 4.92 & 4.93 & 4.96 \\
\hline $2 s^{2} 2 p-2 s^{2} 3 p$ & 6.37 & 5.57 & 6.09 & 5.99 & 6.02 \\
\hline $2 s^{2} 2 p-2 s^{2} 3 d$ & 7.00 & 6.15 & 6.71 & 6.76 & 6.79 \\
\hline $2 s^{2} 2 p-2 s^{2} 4 s$ & 7.02 & 6.34 & 6.89 & 6.78 & 6.82 \\
\hline
\end{tabular}

${ }^{a}$ Ref. 37, ${ }^{b}$ Ref. 31.

TABLE IV: $\mathrm{Na}_{3}$ : GW, GWГ and LGW results (without BSE) for the PAEs compared with the previous MRDCI[38] and full CI[40] results and experimental data[35] in units of $\mathrm{eV}$

\begin{tabular}{ccccccc}
\hline \hline Transition & GW & GW & LGW & MRDCI $^{\mathrm{a}}$ & FCI $^{\mathrm{b}}$ & Expt. $^{\mathrm{c}}$ \\
\hline${ }^{2} \mathrm{~B}_{2}-1^{2} \mathrm{~A}_{1}$ & 0.75 & 0.77 & 0.80 & 0.52 & 0.48 & \\
\hline${ }^{2} \mathrm{~B}_{2}-2^{2} \mathrm{~B}_{1}$ & 1.14 & 1.14 & 1.10 & - & - & \\
\hline${ }^{2} \mathrm{~B}_{2}-2^{2} \mathrm{~A}_{1}$ & 1.21 & 1.34 & 1.34 & 1.07 & 1.09 & \\
\hline${ }^{2} \mathrm{~B}_{2}-2^{2} \mathrm{~B}_{2}$ & 1.43 & 1.69 & 1.67 & 1.33 & - & \\
\hline${ }^{2} \mathrm{~B}_{2}-1^{2} \mathrm{~A}_{2}$ & 1.71 & 1.92 & 1.94 & 1.77 & - & 1.85 \\
\hline${ }^{2} \mathrm{~B}_{2}-4^{2} \mathrm{~A}_{1}$ & 1.96 & 1.99 & 2.12 & 1.97 & 1.96 & 2.02 \\
\hline${ }^{2} \mathrm{~B}_{2}-3^{2} \mathrm{~B}_{1}$ & 2.34 & 2.36 & 2.50 & - & - & \\
\hline${ }^{2} \mathrm{~B}_{2}-2^{2} \mathrm{~A}_{2}$ & 2.55 & 2.38 & 2.56 & 2.61 & 2.51 & 2.58 \\
\hline${ }^{2} \mathrm{~B}_{2}-6^{2} \mathrm{~B}_{2}$ & 3.04 & 2.56 & 2.60 & 2.85 & - & \\
\hline \hline
\end{tabular}

${ }^{a}$ Ref. 38, ${ }^{b}$ Ref. 40, ${ }^{c}$ Ref. 35.

The LGW $\Gamma$ results are roughly the same or better accuracy compared with the QC calculations; see also Fig. 2. However, the non-linearized GW results for B atom and, in particular, higher excitations for $\mathrm{Na}_{3}$ and $\mathrm{Li}_{3}$ are not very good. This disagreement is caused by the wide gap between the QP energies at the HOMO and LUMO levels. In the case of narrow gap systems, i.e. when the HOMO and LUMO QP energies are not much different, approximating the energy dependence of the self-energy by substitutiing the HOMO QP energy is a good approximation. On the other hand, if the LUMO QP energy is much higher than the HOMO QP energy, the approximation is no longer valid and should be improved by using the linearized LGW method as shown here.

TABLE V: $\mathrm{Li}_{3}$ : GW, GW and $\mathrm{LGW \Gamma}$ results (without BSE) for the PAEs compared with the previous MRDCI and estimated full CI (esFCI) results[39] and experimental data[36, 41] in units of eV

\begin{tabular}{ccccccc}
\hline \hline Transition & GW & GW & LGW & MRDCI $^{\mathrm{a}}$ & esFCI & Expt. \\
\hline${ }^{2} \mathrm{~B}_{2}-1^{2} \mathrm{~A}_{1}$ & 0.32 & 0.34 & 0.36 & 0.317 & 0.216 & \\
\hline${ }^{2} \mathrm{~B}_{2}-1^{2} \mathrm{~B}_{1}$ & 0.71 & 0.73 & 0.71 & 0.787 & 0.711 & \\
\hline${ }^{2} \mathrm{~B}_{2}-2^{2} \mathrm{~A}_{1}$ & 1.16 & 1.19 & 1.25 & 1.206 & 1.136 & \\
\hline${ }^{2} \mathrm{~B}_{2}-2^{2} \mathrm{~B}_{2}$ & 1.33 & 1.63 & 1.67 & 1.430 & 1.346 & \\
\hline${ }^{2} \mathrm{~B}_{2}-3^{2} \mathrm{~A}_{1}$ & 1.64 & 1.80 & 1.89 & 1.612 & 1.498 & $1.81^{\mathrm{b}}$ \\
\hline${ }^{2} \mathrm{~B}_{2}-1^{2} \mathrm{~A}_{2}$ & 1.77 & 2.08 & 2.18 & 1.975 & 1.937 & \\
\hline${ }^{2} \mathrm{~B}_{2}-2^{2} \mathrm{~B}_{1}$ & 2.22 & 2.32 & 2.47 & 2.320 & 2.245 & \\
\hline${ }^{2} \mathrm{~B}_{2}-3^{2} \mathrm{~B}_{2}$ & 2.73 & 2.43 & 2.63 & 2.615 & 2.407 & $2.61^{\mathrm{c}}$ \\
\hline \hline
\end{tabular}

${ }^{a}$ Ref. 39, ${ }^{b}$ Ref. 36, ${ }^{c}$ Ref. 41.

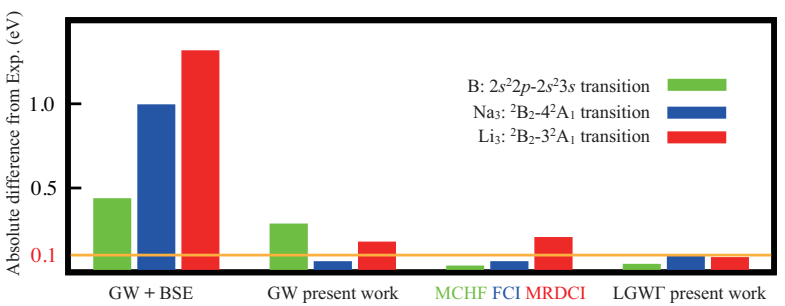

FIG. 2: Comparison of PAEs calculated by GW + BSE, GW present work (without BSE), MCHF (for B), FCI (for $\mathrm{Na}_{3}$ ), MRDCI (for $\mathrm{Li}_{3}$ ), and LGW $\Gamma$ present work (without BSE). Green, blue, red bars represent, respectively, the deviations of the calculated PAEs from the experimental values for $\mathrm{B}, \mathrm{Na}_{3}$, and $\mathrm{Li}_{3}$. The $\mathrm{GW}$ present work greatly improves the GW + BSE result but most of the deviation is still more than $0.1 \mathrm{eV}$. The LGW present work shows excellent agreement with the experimental value within $0.1 \mathrm{eV}$, while the MRDCI result for $\mathrm{Li}_{3}$ shows $>0.1 \mathrm{eV}$ deviation. Experimental values as well as MCHF, MRDCI, and FCI values are taken from Refs. 31, 35-40

\section{CONCLUSION}

In this paper, we proposed the $\mathrm{GW}(\Gamma)$ method without BSE to calculate photoabsorption energies (PAEs). This method is in particular useful to spin-polarized systems. As a result, this method yields good agreement with the experimentally observed PAEs for $\mathrm{Al}, \mathrm{B}, \mathrm{Na}_{3}$, and $\mathrm{Li}_{3}$. The results are much better than those of the GW + BSE method. The result for $\mathrm{Al}$ is already excellent within the $0.1 \mathrm{eV}$ difference from the experimental value. We also applied the beyond GW methods without BSE such as the self-consistent GW $\Gamma$ and (linearized) LGW $\Gamma$ methods, and found that the LGW BSE yields excellent agreement with the available experimental data within $0.1 \mathrm{eV}$ for $\mathrm{B}, \mathrm{Na}_{3}$, and $\mathrm{Li}_{3}$. Our non-linearized 
GWT results (without BSE) suggest that, in the calculation of the QP energies much higher than the HOMO energy, the energy dependence of the self-energy becomes a very important problem. The linearization of the energy dependence is essential to avoid this problem. The best is to use the self-consistent (linearized) LGW method including the vertex correction. Its computational cost scales as $O\left(N^{2} M^{3}\right)$, where $N$ and $M$ are the numbers of basis functions and empty states, respectively, if the plasmon-pole model[28] is used for the $\Gamma$-related calculations (full frequency integration can be used for the GWrelated calculations)[16]. This is comparable to the MRDCI method, but much less expensive than the full CI calculations. It is left for the future study to apply this method to larger molecules.

\section{Acknowledgments}

This work was supported in part by the Ministry of Education, Culture, Sports, Science and Technology (MEXT) Japan as a social and scientific priority issue (Creation of new functional devices and high-performance materials to support next-generation industries) to be tackled by using post-K computer for the use of the supercomputer facilities of the Supercomputer Centers at the Institute for Solid State Physics, the University of Tokyo, at Hokkaido University, and at IMR, Tohoku University (Project IDs. hp160072, hp160234, hp170268, and hp170190).
[1] A. Szabo, and N. S. Ostlund, Modern Quantum Chemistry: Introduction to Advanced Electronic Structure Theory (Dover, New York, 1996).

[2] E. Runge and E. K. U. Gross, Phys. Rev. Lett. 52, 997 (1984).

[3] G. Onida, L. Reining, R. W. Godby, R. Del Sole, and W. Andreoni, Phys. Rev. Lett. 75, 818 (1995).

[4] S. Albrecht, L. Reining, R. Del Sole, and G. Onida, Phys. Rev. Lett. 80, 4510 (1998).

[5] M. Rohlfing and S. G. Louie, Phys. Rev. Lett. 80, 3320 (1998).

[6] M. Rohlfing and S. G. Louie, Phys. Rev. B 62, 4927 (2000).

[7] G. Onida, L. Reining, and A. Rubio, Rev. Mod. Phys. 74, 601 (2002).

[8] D. B. Krisiloff and E. A. Carter, Phys. Chem. Chem. Phys. 14, 7710 (2012)

[9] V. N. Glushkov and X. Assfeld, Theor. Chem. Acc. 135, 3 (2015).

[10] D. Jacquemin, I. Duchemin, and X. Blase, J. Chem. Theory Comput. 11, 3290 (2015).

[11] L. Hung, F. Bruneval, K. Baishya, and S. Öğt, J. Chem. Theory Comput. 13, 2135 (2017).

[12] M. S. Hybertsen and S. G. Louie, Phys. Rev. B 34, 5390 (1986).

[13] R. W. Godby, M. Schlüter, and L. J. Sham, Phys. Rev. B 37, 10159 (1988).

[14] D. Hirose, Y. Noguchi, and O. Sugino, Phys. Rev. B 91, 205111 (2015).

[15] D. Jacquemin, I. Duchemin, X. Blase, J. Chem. Theory Comput. 11, 3290 (2015).

[16] R. Kuwahara, Y. Noguchi, and K. Ohno, Phys. Rev. B 94, 121116(R) (2016).

[17] F. Bruneval, S. M. Hamed, and J. B. Neaton, J. Chem. Phys. 142, 244101 (2015).

[18] K. Ohno, S. Ono, and T. Isobe, J. Chem. Phys. 146, 084108 (2017).

[19] J. Bois and T. Körzdörfer, J. Chem. Theory Comput. 13, 4962 (2017).

[20] T. Kotani, M. van Schilfgaarde, and S. V. Faleev, Phys. Rev. B 76, 165106 (2007).

[21] M. Shishkin, M. Marsman, and G. Kresse, Phys. Rev. Lett. 99,
246403 (2007).

[22] X. Ren, P. Rinke, G. E. Scuseria, and M. Scheffler, Phys. Rev. B 88, 035120 (2013).

[23] L. Hung, F. H. da Jornada, J. Souto-Casares, J. R. Chelikowsky, S. G. Louie, and S. Ögüü, Phys. Rev. B 94, 085125 (2016).

[24] A. Grüneis, G. Kresse, Y. Hinuma, and F. Oba, Phys. Rev. Lett. 112, 096401 (2014).

[25] E. Maggio and G. Kresse, J. Chem. Theory Comput. 13, 4765?4778 (2017).

[26] R. Kuwahara and K. Ohno, Phys. Rev. A. 90, 032506 (2014).

[27] S. Ono, Y. Noguchi, R. Sahara, Y. Kawazoe, and K. Ohno, Computer Phys. Commun. 189, 20 (2015).

[28] W. von der Linden and P. Horsch, Phys. Rev. B 37, 8351 (1988).

[29] F. Bruneval, Phys. Rev. Lett. 103, 176403 (2009).

[30] K. Victor and W. C. Martin, J. Phys. Chem. Ref. Data 20, 775 (1991)

[31] A. E. Kramida and A. N. Ryabtsev, Phys. Scr. 76, 544 (2007).

[32] A. Herrmann, S. Leutwyler, E. Schumacher, and L. Wöste, Helv. Chim. Acta 61, 453 (1978).

[33] Ph Dugourd, D. Rayane, P. Labastie, B. Vezin, J. Chevaleyre, and M. Broyer, Chem. Phys. lett. 197, 433 (1992).

[34] W. C. Martin and R. Zalubas, J. Phys. Chem. Ref. Data 8, 817 (1979)

[35] C. R. C. Wang, S. Pollack, T. A. Dahlseid, G. M. Koretsky, and M. M. Kappes, J. Chem. Phys. 96, 7931 (1992).

[36] H.-G. Krämer, M. Keil, C. B. Suarez, W. Demtröder, and W. Meyer, Chem. Phys. Lett. 299, 212 (1999).

[37] H. Lundberg, Z. S. Li, and P. Jönsson, Phys. Rev. A 63, 032505 (2001).

[38] V. Bonačićkoutecký, P. Fantucci, and J. Koutecký, J. Chem. Phys. 93, 3802 (1990).

[39] H. O. Beckmann, Chem. Phys. Lett. 93, 240 (1982).

[40] P. K. Priya, D. K. Rai, and A. Shukla, Eur. Phys. J. D 71, 116 (2017).

[41] Ph. Dugourd, J. Chevaleyre, M. Broyer, J. P. Wolf, and L. Wöste”, Chem. Phys. Lett. 175, 555 (1990). 